\title{
Dynamics of the transition from polarization disorder to antiphase polarization domains in vector fiber lasers
}

\author{
Caroline Lecaplain, ${ }^{1}$ Philippe Grelu, ${ }^{1}$ and Stefan Wabnitz ${ }^{2, *}$ \\ ${ }^{1}$ Laboratoire Interdisciplinaire Carnot de Bourgogne, UMR 6303 CNRS, Université de Bourgogne, 9 av. Alain Savary, 21078 Dijon, France \\ ${ }^{2}$ Dipartimento di Ingegneria dell'Informazione, Università di Brescia, Via Branze 38, Brescia 25123, Italy
}

(Received 17 December 2013; published 16 June 2014)

\begin{abstract}
We demonstrate that nonlinear polarization coupling in a fiber ring laser without polarization-selective elements, subject to the effects of average anomalous dispersion, Kerr effect, and nonlinear gain saturation, can lead to the antisynchronization of spatiotemporal chaos into a wide variety of ordered laminar states of orthogonal polarization temporal domains. These antiphase polarization domains include stable lattices of soliton trains with high duty cycle at repetition rates of hundreds of $\mathrm{MHz}$, as well as sparse trains of coupled dark and bright solitary waves.
\end{abstract}

DOI: 10.1103/PhysRevA.89.063812

PACS number(s): 42.65.Sf, 42.55.Wd, 42.81.Gs, 05.45.Xt

\section{INTRODUCTION}

The synchronization of individual chaotic nonlinear oscillators by means of their coupling is a subject of great interest in several domains of physical sciences and engineering applications [1,2]. In particular, by adjusting the coupling among the oscillators one may control and stabilize otherwise chaotic physical systems and devices, which extends in a significant way the well-known effect of synchronization of regular oscillators [3]. Although the synchronization of chaos has been extensively investigated over the past 20 years, and has found numerous applications such as secure communication systems [4,5], the demonstrated examples have been mostly restrained to the case of discrete oscillators, whose evolution is described by ordinary differential equations. Much less is known for extended spatiotemporal chaotic physical systems described in terms of partial differential equations. In this case, one may invoke thermodynamic analogies allowing to classify the numerically found regions of chaotic or ordered behavior in terms of phase diagrams and transitions [6].

In this work, we demonstrate a relatively simple example of spatiotemporal synchronization, which occurs in the polarization state of a ring cavity fiber laser built without polarizationdependent-loss elements, so that full vector propagation is allowed [7]. Spatiotemporal propagation of the vector optical field in the laser is subject to average anomalous chromatic dispersion, the Kerr effect, and nonlinear gain saturation. As we shall see, the experiments demonstrate the transition from spatiotemporal polarization disorder into ordered laminar states of orthogonal polarization temporal domains, including stable lattices of soliton trains with high duty cycle and hundreds of $\mathrm{MHz}$ repetition rates, as well as trains of coupled hole-pulse (or dark and bright) solitary waves.

The richness of the observed behavior may be qualitatively well reproduced in terms of a minimal model for vector dissipative structures, which is provided by the coupled complex Ginzburg-Landau equations (CGLE) [8-12]. As is well known, the scalar and vector GLEs describe pattern formation phenomena in a variety of nonequilibrium systems near a Hopf bifurcation, such as phase transitions [13,14],

*stefan.wabnitz@unibs.it superconductivity, superfluidity, Bose-Einstein condensation, field theory, Bénard convection [15], Taylor-Couette flow between rotating cylinders [16], plane Poiseuille flow [17], drift waves in plasmas, chemical reactions and turbulence [18], ionization waves [19], liquid crystals [20], biophysical systems [21] and laser devices [22-24].

In Sec. II, we provide a detailed justification for the use of the coupled CGLE equations as our minimal laser model. Then, by exploring the equation parameters, we present numerical simulations showing the formation of a diversity of laminar states with antiphase dynamics between orthogonal polarization components. In Sec. III, we report our experimental observations performed with an erbium-doped fiber ring laser cavity, highlighting the strong analogies with the simulated dynamics. Finally, we provide a synthesis of our understanding of the vector dynamics in the concluding Sec. IV.

\section{MODELING VECTOR DYNAMICS WITH COUPLED GINZBURG-LANDAU EQUATIONS}

The diversity of the temporal dynamics of the light field emerging from fiber ring lasers is challenging models developed to date. Resorting to overly complex numerical simulations with too many uncertain parameters may hamper the general classification of a dynamics that appears to be universal, being observed in a variety of experimental setups. We here consider active fiber ring cavities that allow for the propagation and coupling of both polarization components, in the absence of an obvious saturable absorption mechanism. This excludes, for instance, the use of a polarization sensitive isolator, which freezes one polarization degree of freedom, while at the same time it may trigger passive mode locking through nonlinear polarization evolution in the optical fibers. Instead, by assuming a low level of polarization-dependent losses, the coupling between the two polarization components takes place throughout the entire cavity length, and it can be tuned by means of discrete wave plates, or lossless polarization controllers. By playing with the orientation of these wave plates, a diversity of vector pulsations can be observed [7,25-28]. 
The most striking observations comprise antiphase polarization dynamics. Some of these antiphase pulses have recently been interpreted as polarization-domain-wall (PDW) complexes [7,27]. The formation of PDWs in the normal path-averaged dispersion regime was analytically reproduced in terms of the PDW solutions of a purely conservative Hamiltonian model based on two incoherently coupled nonlinear Schrödinger (NLS) equations [7,29,30]. In Ref. [7], the noise-generated spontaneous emergence of PDWs was also numerically confirmed by the full numerical simulations of field propagation in the various cavity components, including gain saturation in the fiber amplifier.

We report here the observation of similar structures in the anomalous dispersion propagation regime as well. In addition to the PDWs, in the anomalous dispersion regime we have also observed the spontaneous emergence of a rich variety of temporal structures, involving a large diversity of square pulse durations and repetition rates. Finally, the observed stability of these structures which are spontaneously generated from amplified spontaneous emission noise, and are repeatedly monitored round trip after round trip for an almost unlimited time, contradicts with the marginal stability of the PDWs' structures, which are predicted in the frame of purely conservative models. Clearly, the diversity of temporal dynamics that is accessible by simply adjusting the intracavity wave plates, and the stability that is observed once that the wave-plate parameters are fixed, are the signature of the inherently dissipative origin of the observed nonlinear structures.

Essential dissipative effects comprise gain saturation, which prevents energy blowup, and bandpass filtering, which localizes the solution in the spectral domain. It is also known that cross-gain saturation between two laser modes favors the appearance of antiphase pulsations [25,31-36]. These dissipative terms, accompanied by chromatic dispersion as well as self- and crossed-Kerr nonlinearities, make up one of the simplest vector model for dissipative nonlinear systems, namely the vector-CGLE.

Just before laying down our phenomenological model, let us remark that the pulsation rates reported in Sec. III are fundamentally self-starting, and embedded into the cavity round-trip frequency. These features are obviously beyond the scope of our distributed model. Indeed, whereas it is well known that CGLE models can describe a variety of pulsed and mode-locked dynamics [24], modeling self-starting requires the inclusion of a noise source, and the cavity round-trip periodicity implies the loss of translational symmetry. We conjecture that the early stage of the development of periodic pulsations may be triggered by the Risken-NummedalGraham-Haken (RNGH) instability [37,38]. At high pumping levels, which is always the case experimentally, Rabi splitting induces additional gain for adjacent cavity modes, which start to oscillate and beat at the fundamental cavity frequency. The RNGH instability finds suitable conditions to develop in fiber lasers due to the relatively long cavity lengths, which entail small intermode spacings, as well as to the large gain and loss contributions per cavity round-trip.

The minimal model for the emergence of vector dissipative structures in fiber lasers is provided, in the mean-field approximation and in dimensionless units, by the following

\section{CGLE:}

$$
\begin{aligned}
& \partial_{t} U=U+(1+i \beta) \partial_{\tau}^{2} U-(1-i \alpha)|U|^{2} U-(\gamma-i \rho)|V|^{2} U, \\
& \partial_{t} V=V+(1+i \beta) \partial_{\tau}^{2} V-(1-i \alpha)|V|^{2} V-(\gamma-i \rho)|U|^{2} V,
\end{aligned}
$$

where $U(t, \tau)$ and $V(t, \tau)$ represent the two orthogonal polarization components of the field in the cavity. The independent variables $t$ and $\tau$ are the slow and fast temporal coordinates that describe field variations on the slow time scale of the cavity round-trip time and on the fast time scale associated with bandpass filtering and chromatic dispersion, respectively. The first term on the right-hand side of Eq. (1) represents smallsignal linear gain; the first contribution to the second term describes bandwidth limited gain, and $\beta$ is associated to group velocity dispersion (GVD). Positive (negative) $\beta$ corresponds to anomalous (normal) GVD. The first contribution to the third term in the right-hand side of Eq. (1) represents the nonlinear saturation of gain, whereas $\alpha$ is the nonlinear Kerr effect. For the self-focusing nonlinearity of silica fibers, one has $\alpha>0$. Whereas $\gamma$ and $\rho$ describe nonlinear polarization cross-gain saturation and rotation, respectively.

An important issue with dissipative effects is their finite response time. For a gain medium such as an erbium-doped glass fiber, the population relaxation time is particularly long, in the range of $10 \mathrm{~ms}$. However, excited-state absorption and ion-pair interaction are known to produce much faster nonlinear loss contributions [39]. In addition, it has been pointed out that multiple passes through optical amplifiers, despite the slow transient responses of the individual element, are able to trigger fast transients as a result of cascading of the slow transient response of the individual amplifier [40]. In a cavity, such a phenomenon is fully exacerbated, so that slow dynamics such as gain transient effects are known to significantly affect the formation of ultrashort pulse structures down to the picosecond time range [41]. Gain saturation alone has been used to explain self mode locking with nanosecond pulse formation [42]. It is therefore essential not to confuse the buildup time of structures, which can take thousands of cavity round-trips, with the duration of typical pulse structures. Therefore, we assume that at the leading-order effective instantaneous saturation terms can be incorporated into the previously introduced minimal model of Eq. (1).

Equation (1) has the $\tau$-independent or continuous wave (cw) solutions

$$
U(t, \tau)=U_{0} \exp ^{i K_{U} t} ; \quad V(t, \tau)=V_{0} \exp ^{i K_{V} t},
$$

where $K_{U}=\alpha U_{0}^{2}+\rho V_{0}^{2}$ and $K_{V}=\alpha V_{0}^{2}+\rho U_{0}^{2}$. In the absence of nonlinear polarization coupling (i.e., with $\gamma=\rho=0$ ), one obtains two independent Ginzburg-Landau equations whose attractor is provided by the field amplitudes $U_{0}=$ $V_{0}=1$. In this case, it is known that solutions of the type (2) are stable whenever the Benjamin-Feir (BF) condition $1-\alpha \beta>0$ is satisfied. Otherwise, whenever the BF condition is violated one observes different types of chaotic behaviors in solutions of the uncoupled Eq. (1) (phase and defect chaos, see Ref. [43]). Even in the BF stable case, it has been observed that the cw solutions coexist with a spatiotemporal 

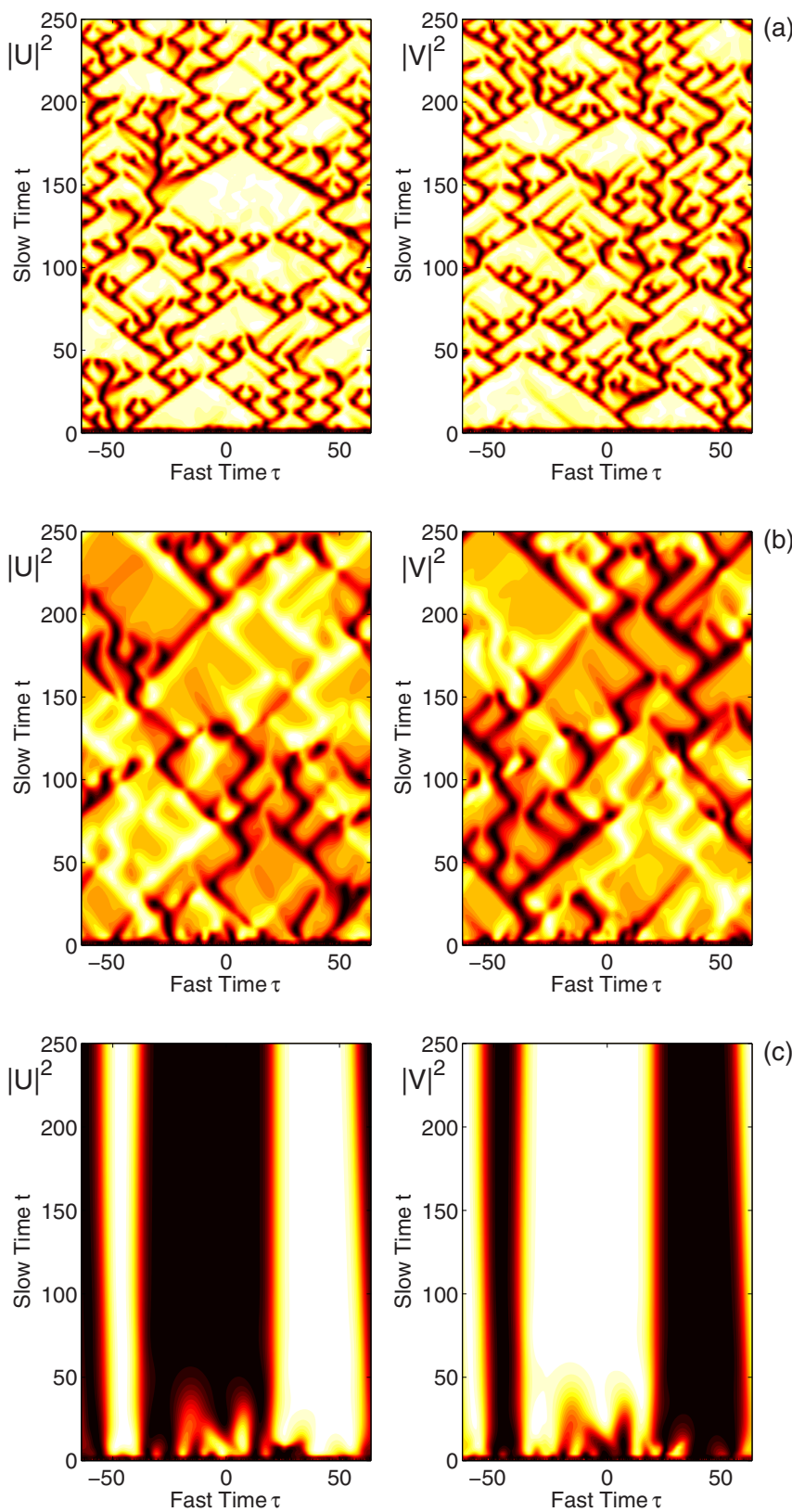

FIG. 1. (Color online) Contour plots showing, in dimensionless units, the evolution of intensities $|U(t, \tau)|^{2}$ (left column) and $|V(t, \tau)|^{2}$ (right column) of the two polarization components vs. fast time $\tau$ and slow time $t$, respectively, for different nonlinear polarization coupling coefficients $\gamma$ : (a) $\gamma=0.1$; (b) $\gamma=0.6$; (c) $\gamma=1.05$. Other parameters are $\alpha=2, \beta=0.2$, and $\rho=\alpha \gamma$.

chaotic attractor, a phenomenon which has been described as spatiotemporal intermittency (STI) [6]. The corresponding intermittent behavior is the same which is observed in the numerical solution of the CGLE (1) for relatively weak polarization coupling (e.g., with $\gamma=0.1$ and $\rho=\alpha \gamma$ ), see Fig. 1(a). In our numerical simulations, we have solved Eq. (1) with the split-step Fourier method involving $N=512-1024$ modes, using a typical integration step $d t=10^{-3}$ and the periodic boundary conditions $U(t, \tau=-T / 2)=U(t, \tau=T / 2)$ and $V(t, \tau=-T / 2)=V(t, \tau=T / 2)$, where $T=128-256$.
As an initial condition, unless otherwise specified, we used for both polarizations independent complex white noise seeds: both real and imaginary components of the noise field had a normal distribution with variance $\sigma^{2}=0.05$.

The presence of sufficiently strong polarization coupling permits to substantially change the behavior of STI [8-11]. Indeed, for weak polarization coupling $\gamma \ll 1$, the cw solution (2) with $U_{0}^{2}=V_{0}^{2}=1 /(1+\gamma)$ is stable whenever the $\mathrm{BF}$ condition $1-\alpha \beta>0$ is verified; whereas the uncoupled solutions where either $U_{0}=0$ or $V_{0}=0$ are unstable. Indeed, Fig. 1(a) shows that, whenever $\gamma=0.1$, the $U$ and $V$ polarization components follow fully independent chaotic dynamics: see also the intensity profiles which are extracted at $t=250$ in Fig. 2(a). We also notice large transient bright regions in Fig. 1(a). These regions correspond to a nearly uniform polarization in laminar domains that spontaneously form, then gradually disappear as a result of moving opposite fronts, hence the peculiar triangular-shaped domains displayed on the contour plot figures. The moving fronts are associated with the propagation of the so-called homoclinic holes, which are generated by phase slip events [44].

As the polarization coupling grows larger, it is interesting to notice that the polarization dynamics of the $U$ and $V$ components displays a clear anticorrelation or antiphase behavior: see, for example, Fig. 1(b), where $\gamma=0.6$. Indeed, in Fig. 1(b) the dark hole-like traveling structures in one polarization component are exactly superimposed with bright pulse-like laminar structures in the orthogonal polarization. The anticorrelation behavior which is present even in the spatiotemporal intermittence dynamics is clearly shown by the corresponding intensity plots that are extracted for $t=250$ in Fig. 2(b). A more precise quantitative description of the transition from chaotic to anti-correlated behavior in the numerical solutions of the two components of Eq. (1) in terms of joint probability distributions, with associated mutual information and correlation coefficients, can be found in previous literature [9].

Thus one obtains an antisymmetric coupled STI regime as the result of the synchronization of spatiotemporal chaos. As the polarization cross-coupling $\gamma$ grows up to values right below unity (e.g., for $\gamma=0.95$ ), the simulations show that the anticorrelated STI regime has a metastable behavior. Indeed, it is only after a relatively large evolution time such as $t \cong$ 300 that STI decays into the stable cw solution with identical amplitude for the two polarization components $U_{0}^{2}=V_{0}^{2}=$ $1 /(1+\gamma)$.

On the other hand, for relatively large polarization couplings, namely for $\gamma>1$, there is only one stable polarization component that emerges from their competition: a stable solution of Eq. (1) requires that either $U_{0}=1, V_{0}=0$ or $U_{0}=$ $0, V_{0}=1$. In fact we may extend the cw solution (2) to include a finite frequency shift for each polarization component, say, $\Omega_{U, V}$, as well as a $t$ dependence of the $U_{0}$ and $V_{0}$ amplitudes

$U(t, \tau)=U_{0}(t) \exp ^{i\left(K_{U} t-\Omega_{U} \tau\right)} ; \quad V(t, \tau)=V_{0}(t) \exp ^{i\left(K_{V} t-\Omega_{V} \tau\right)}$.

By substituting Eq. (2) into Eq. (1), one obtains the coupled ordinary differential equations (ODEs) for the polarization 
(a)

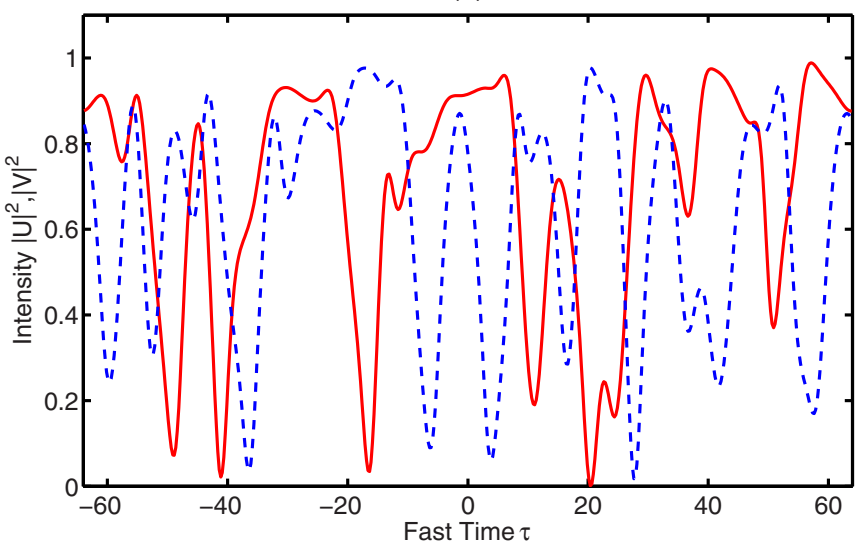

(b)

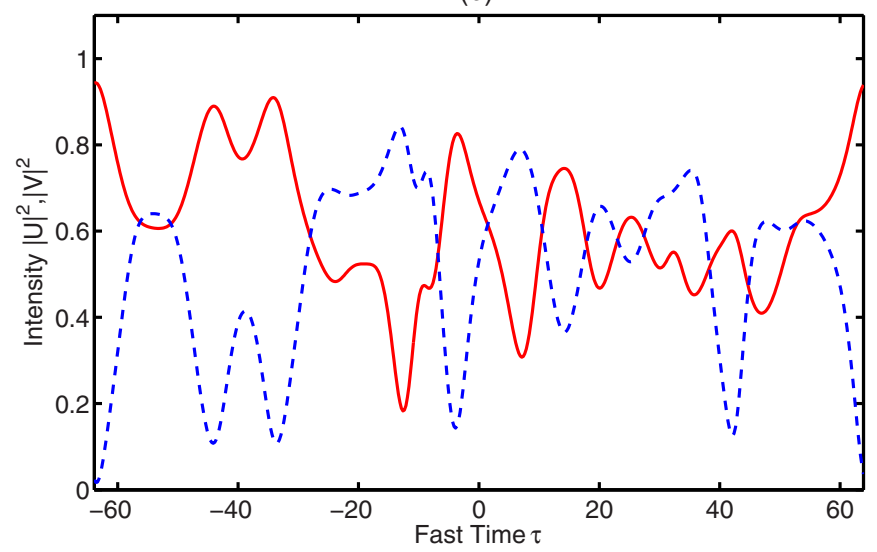

(c)

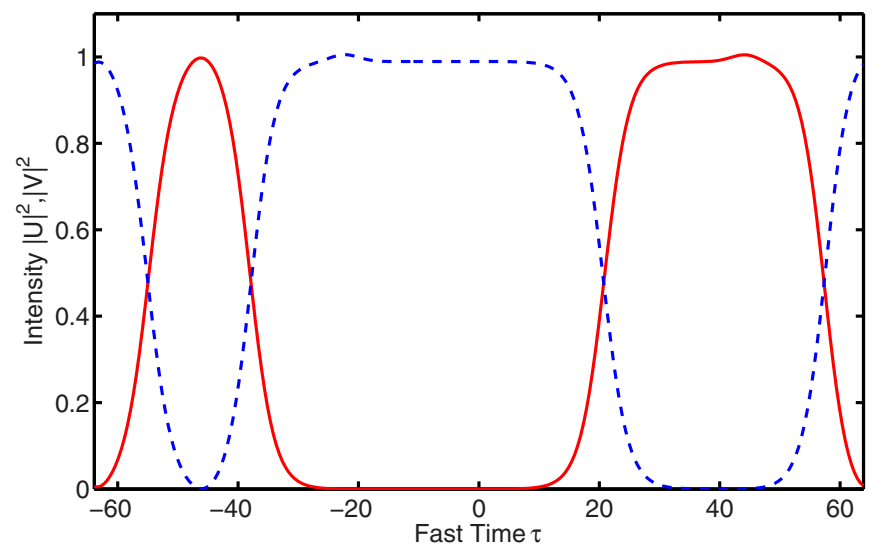

FIG. 2. (Color online) Intensity profiles, in dimensionless units, of $|U(t, \tau)|^{2}$ (red solid curves) and $|V(t, \tau)|^{2}$ (dashed blue curves) of the two polarization components vs. $\tau$, for different nonlinear polarization coupling coefficients $\gamma$ at $t=250$ : (a) $\gamma=0.1$; (b) $\gamma=$ 0.6 ; (c) $\gamma=1.05$. Other parameters are $\alpha=2, \beta=0.2$, and $\rho=\alpha \gamma$.

amplitudes

$$
\begin{aligned}
& \partial_{t} U_{0}=\left(1-\Omega_{U}^{2}-U_{0}^{2}-\gamma V_{0}^{2}\right) U_{0}, \\
& \partial_{t} V_{0}=\left(1-\Omega_{V}^{2}-V_{0}^{2}-\gamma U_{0}^{2}\right) V_{0} .
\end{aligned}
$$

By supposing, for example, that $U_{0}=0, \Omega_{U}=0$, and $V_{0} \neq 0$, then from Eq. (4) one obtains that the $U_{0}$ mode has the effective growth rate $g_{U}=1-\gamma V_{0}^{2}=1-\gamma\left(1-\Omega_{V}^{2}\right)$. The stability of the $V_{0}$ polarization requires that the growth rate of the competing $U_{0}$ mode $g_{U}<0$. Otherwise a $V_{0}$-polarized wave with frequency $\Omega_{V}$ is unstable whenever $g_{U}>0$, or $\gamma<1 /\left(1-\Omega_{V}^{2}\right)$. Clearly the same reasoning applies to predict the stability of a single polarization state where $V_{0}=0$, $\Omega_{V}=0$, and $U_{0} \neq 0$. In the examples of Fig. 1 , it turns out that for the single polarization domains one has $\left|\Omega_{U, V}\right| \cong 0.1$, so that individual domains are stable for $\gamma>1.01$. On the other hand, the linear stability analysis of Eq. (4) shows that mixed polarization states, that is, states where both $U_{0}$ and $V_{0}$ components are nonzero, are all unstable whenever $\gamma>1$, see Ref. [12].

Indeed, in the numerical simulations of Eq. (1) we observed that, whenever $\gamma=1$, depending on the initial noise seed, after an initial transient only one of the two orthogonal polarization states survives for relatively long times $t$. Whereas as soon as $\gamma>1.01$ (e.g., for $\gamma=1.05$ ), the polarization state switches among the two individual stable states as shown in Figs. 1(c) and 2(c), producing alternating temporal domains of orthogonal polarizations, separated by relatively fast domain-wall-like transitions. These stationary temporal polarization patterns are also known as stable pairs of sources, which are separated by a shock $[9,12]$. The exact shape of the PDW or shock can be well reproduced by inserting the traveling wave ansatz

$$
U(t, \tau)=\hat{U}(\xi) \exp ^{i K_{U} t} ; \quad V(t, \tau)=\hat{V}(\xi) \exp ^{i K_{V} t}
$$

(where $\xi=\tau-t / v$ ) into Eq. (1). This leads to a set of six coupled real ODEs in the single variable $\xi$, which can be integrated by considering orthogonal polarization asymptotic states for $\xi \rightarrow \pm \infty$ : for details, see Ref. [12].

By replacing the white noise seed with a deterministic weak pulse (of different amplitude in each of the two polarization components), one may also obtain from the numerical solution of Eq. (1) the generation of an isolated polarization holepulse pair which resembles a coupled vector bright-and-dark solitary wave structure (see Fig. 3): here the cross-polarization coupling is increased up to $\gamma=1.4$.

It is quite interesting to consider exploring further the parameter space of Eq. (1): Fig. 4 shows that, when operating in the $\mathrm{BF}$ unstable regime (i.e., we have set $\alpha=0.6, \beta=3$, so that $1-\alpha \beta<0, \rho=-3$, and $\gamma=0.95)$, it is also possible to generate a uniform and spatiotemporally stable lattice of anticorrelated (or antiphase) temporal solitons. The individual pulses in each polarization component are well matched by the exact soliton-like solution of the scalar complex GinzburgLandau equation [45], namely,

$$
\begin{aligned}
U(t, \tau) & =U_{0} \operatorname{sech}(K \tau) \exp [i \theta(\tau)-i \Omega t], \\
\theta_{t} & =-Q \tanh (K \tau) .
\end{aligned}
$$

Therefore we may conclude that two stable antiphase periodic soliton trains can be generated in the orthogonal polarization components of the recirculating laser field.

It is interesting to point out that, with the same choice of parameters but in the scalar case of uncoupled polarizations [that is, if set $\rho=\gamma=0$ in Eq. (1)], in spite of the fact that propagation occurs in the $\mathrm{BF}$ unstable regime, no stable generation of soliton trains is observed to emerge from the instability (see Fig. 5). This shows that nonlinear polarization 
(a)
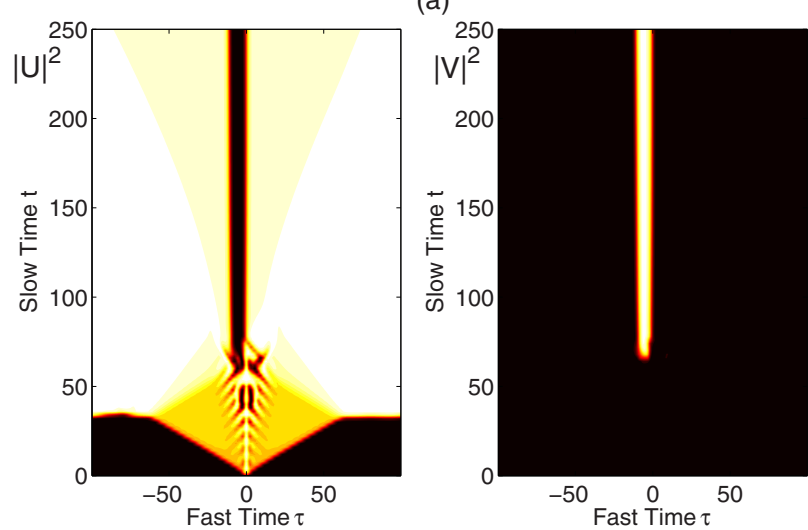

(b)

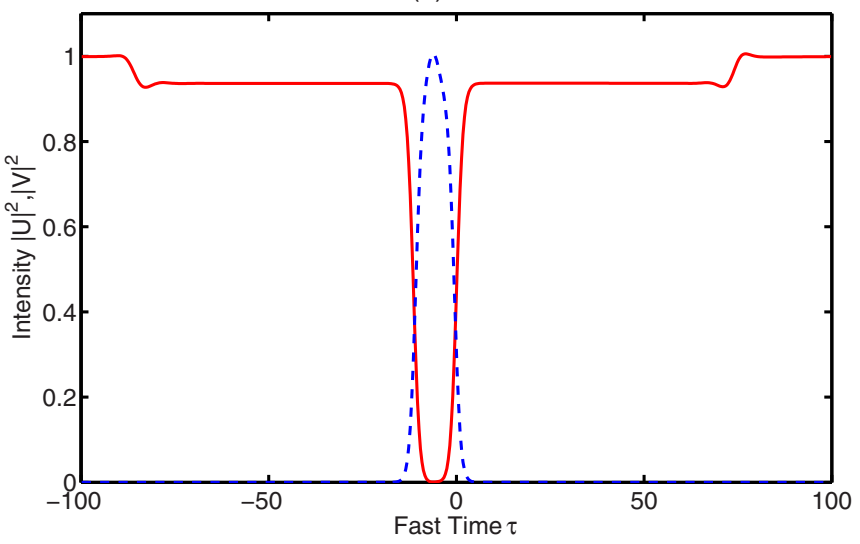

FIG. 3. (Color online) (a) Same as Fig. 1, with $\gamma=1.4$. (b): Intensity profiles of $|U(t, \tau)|^{2}$ (red solid curves) and $|V(t, \tau)|^{2}$ (dashed blue curves) of the two polarization components vs. fast time $\tau$ for the slow time $t=250$.

coupling and cross-gain saturation is indeed essential for creating stable, attracting trains of Pereira-Stenflo solitary waves (6).

\section{EXPERIMENTAL OBSERVATIONS OF VECTOR PATTERNS IN A FIBER RING LASER}

\section{A. Experimental setup}

The experimental setup of the vector fiber laser is displayed in Fig. 6. The laser consists of a ring cavity including a 3-m-long erbium-doped fiber (EDF) which acts as the gain medium and forces its operation at a wavelength $\lambda \approx$ $1.55 \mu \mathrm{m}$. The EDF pumping is provided by a $5-\mathrm{W}$ Raman fiber laser source at $1.48 \mu \mathrm{m}$, coupled through a $1480 / 1550$ multiplexer (WDM). Two additional multiplexers are spliced after the EDF to reject the remaining pumping light from the rest of the cavity, with a suppression ratio better than $30 \mathrm{~dB}$. Unidirectional laser emission is ensured by the presence of two polarization-insensitive optical isolators (ISO) enclosing the amplifier section. Output couplers are placed before $(95 / 5)$ and after (97/3) the amplifier section. The cavity comprises an open-air section, where the polarization of the intracavity light field can be tuned by rotating quarter-wave and half-wave plates. We measured a level of polarization-dependent losses (a)
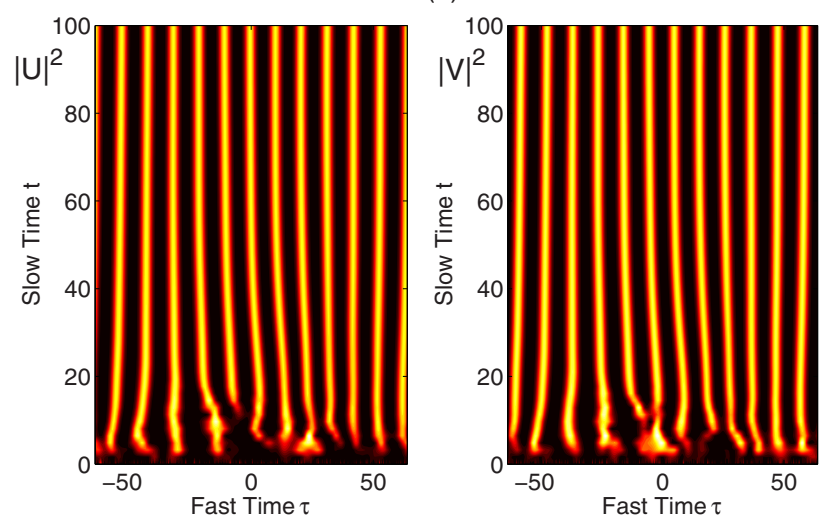

(b)

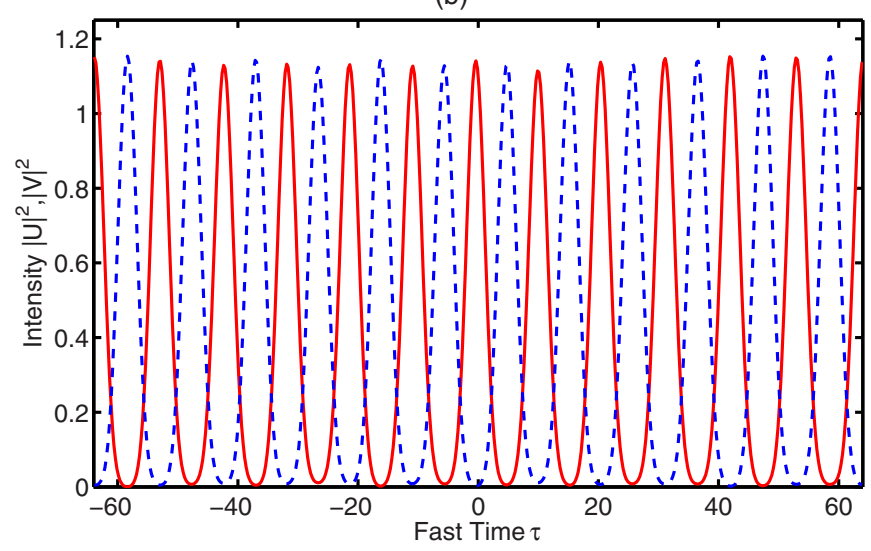

FIG. 4. (Color online) (a) Same as Fig. 1, with $\alpha=0.6, \beta=3$, $\rho=-3$, and $\gamma=0.95$. (b) Intensity profiles of $|U(t, \tau)|^{2}$ (red solid curves) and $|V(t, \tau)|^{2}$ (dashed blue curves) of the two polarization components vs. $\tau$ for $t=100$.

lower than $0.2 \mathrm{~dB}$ over $10 \mathrm{~nm}$ of bandwidth and an average birefringence smaller than $4.10^{-6}$ [7].

The EDF has an anomalous group-velocity dispersion that amounts to $D=+15 \mathrm{ps} \mathrm{nm}^{-1} \mathrm{~km}^{-1}$. Fiber components are pigtailed with short lengths of SMF-28 $(D=$ $+17 \mathrm{ps} \mathrm{nm}^{-1} \mathrm{~km}^{-1}$ ). The cavity also includes a 1.5 -m-long
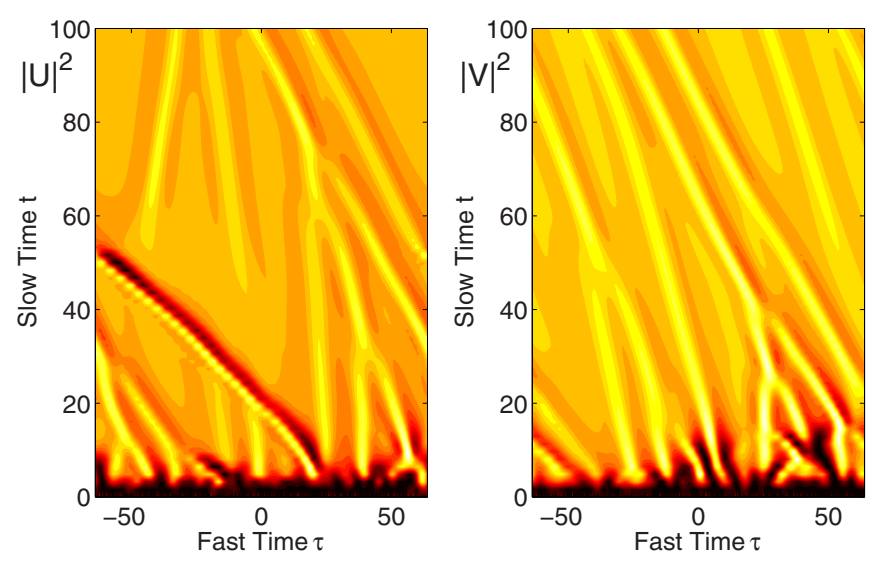

FIG. 5. (Color online) (a) Same as Fig. 4(a), without polarization cross-coupling, i.e., $\gamma=\rho=0$. 


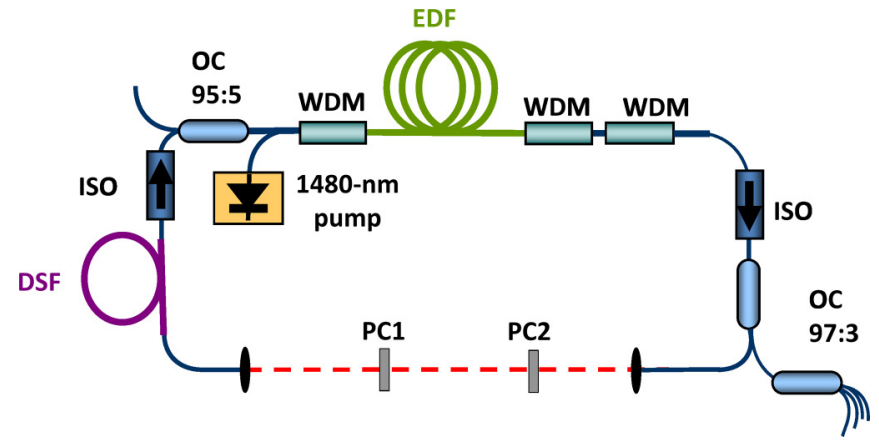

FIG. 6. (Color online) Fiber laser experimental setup. ISO: polarization-insensitive optical isolator, OC: output coupler, EDF: erbium-doped fiber, DSF: dispersion-shifted fiber, PC: quarter- and half-wave plates, WDM: wavelength multiplexer.

dispersion-shifted fiber (DSF, $D=-2.5 \mathrm{ps} \mathrm{nm}^{-1} \mathrm{~km}^{-1}$ ). In contrast to the normal-dispersion case that was studied in Ref. [7], the resulting total cavity chromatic dispersion is anomalous in the present case, and amounts to $-0.013 \mathrm{ps}^{2}$. The fundamental repetition rate of the cavity is $23.9 \mathrm{MHz}$.

The laser signal is monitored using one port of a 1:4 splitter spliced at the $3 \%$ output of the $97 / 3$ coupler. Then, it passes through a combination of quarter- and half-wave plates before it is sent to a polarization beam splitter, which separates the two linear orthogonal polarization components. These polarization components are finally detected by fast photodiodes whose electrical signals are recorded without averaging by a $45-\mathrm{GHz}$, 120-GSa/s real-time oscilloscope. Another output port of the 1:4 splitter is sent to an optical spectrum analyzer, which has a resolution of $65 \mathrm{pm}$.

\section{B. Observation of the transition from disorder to antiphase synchronization}

In the experiment, the most accessible degrees of freedom are the pumping power and the orientation of the intracavity wave plates, which can be tuned while the laser is operated. We found specific regions in the space of the laser parameters where the transition from polarization disorder to antiphase polarization domains could be observed by tuning either the pumping power, or one of the intracavity wave plates. An example of such a transition is provided in Fig. 7, where the pumping power is set at $2 \mathrm{~W}$, and the orientation of the half-wave plate is varied. The situation in Fig. 7(a) exhibits fully independent chaotic temporal dynamics in the two polarizations, as it occurs in the case which is depicted in Fig. 2(a). A sharp transition in the intracavity polarization dynamics from uncorrelated to strongly antiphase correlated polarization evolutions occurs below $\theta=288^{\circ}$. This is corroborated by evaluating the evolution of the Pearson correlation coefficient [see Fig. $7(\mathrm{~g})$ ], which is calculated from the two temporal series of optical intensities (one series for each polarization component), which are simultaneously recorded. The vector antiphase multipulse solitary waves of Fig. 7(e) fill out the entire cavity at a repetition rate of $260 \mathrm{MHz}$. Results very similar to Fig. 7, not shown here, are obtained by varying the pumping power: as the pumping power grows larger, a clear transition from seemingly chaotic, uncorrelated temporal
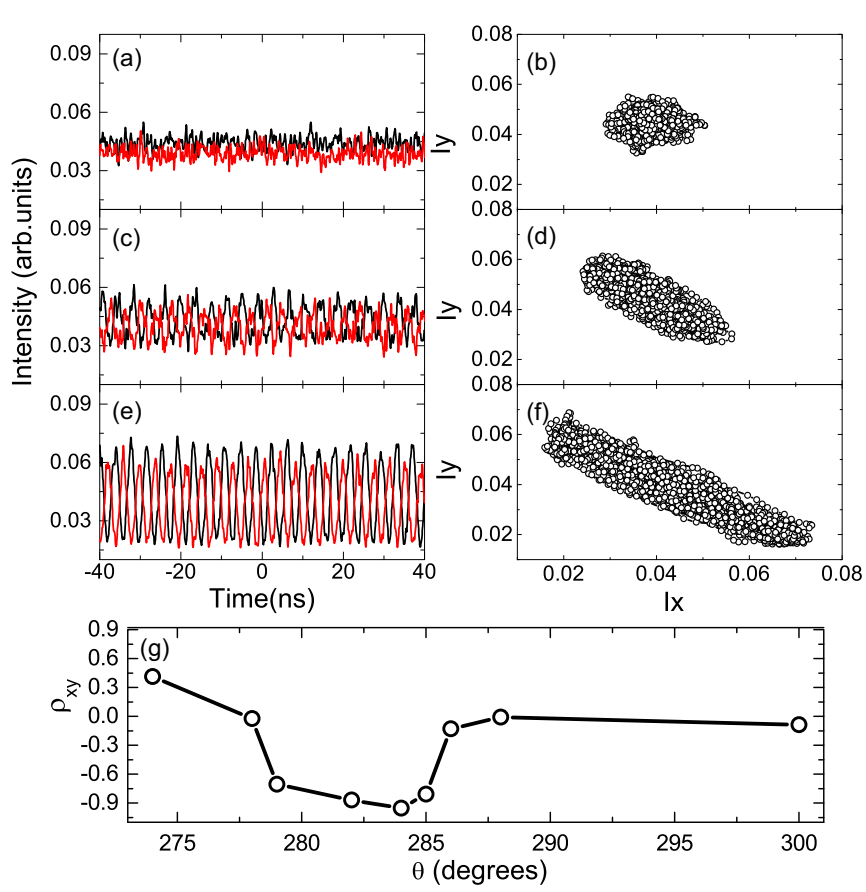

FIG. 7. (Color online) Transition from polarization disorder to antisynchronization, following the orientation $\theta$ of the intracavity half-wave plate. Upper left: Temporal traces of the two orthogonal polarization states. Upper right: Corresponding scatter plots. (a,b) $\theta=288^{\circ}$, (c,d) $\theta=285^{\circ}$, (e,f) $\theta=284^{\circ}$, (g) Pearson correlation coefficient as a function of $\theta$.

fluctuations in both polarizations of the field into antiphase polarization correlated pulse trains is also observed. This demonstrates that the synchronization mechanism is based on nonlinear polarization coupling, as predicted by the CGLE model (1).

To highlight the remarkable stability of the emerging anticorrelated pulse trains, we have recorded the radio-frequency spectrum of the output intensity. In the example shown in Fig. 8, the repetition frequency of these pulse trains reaches $475 \mathrm{MHz}$, namely around 20 times the cavity fundamental repetition rate. Using a radio-frequency spectrum analyzer, we can see in Fig. 9 that the supermode suppression level is as high as than $40 \mathrm{~dB}$ in the $9-\mathrm{GHz}$ span and above $30 \mathrm{~dB}$ in the $1-\mathrm{GHz}$ span.
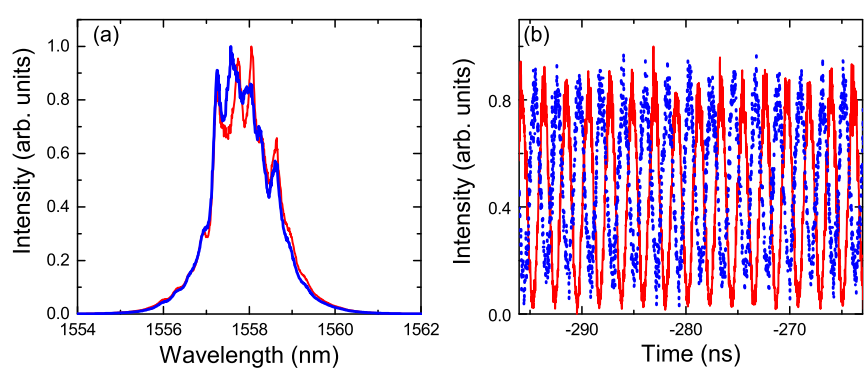

FIG. 8. (Color online) Vector multipulse solitary wave trains filling the cavity at $475 \mathrm{MHz}$. (a) Optical spectra of the two orthogonal polarization states; (b) corresponding temporal traces. 


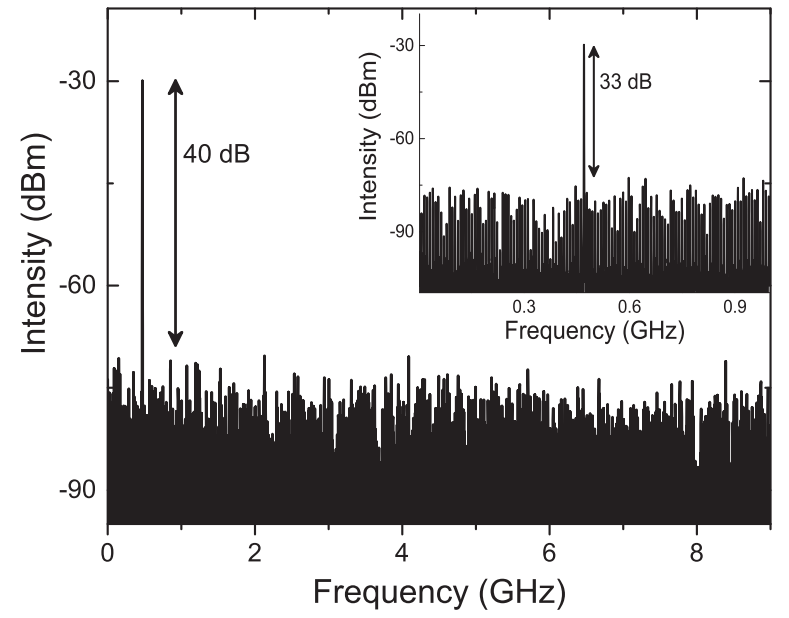

FIG. 9. Radio-frequency analysis of the regime presented in Fig. 8. RF spectra for 9 and $1 \mathrm{GHz}$ (inset) spans.

The pulse trains of Figs. 7(e) and 8 are analogous to the anticorrelated periodic trains of temporal Pereira-Stenflo solitary waves that are depicted in Fig. 4 after numerical solving of the CGLE Eq. (1). In the following, other distinct regimes of transition from polarization disorder to antiphase polarization domains are found, however, the remaining noise level in the antisynchronized states prevented us from obtaining a correlation signature as sharp as in Fig. $7(\mathrm{~g})$.

\section{Coupled vector bright and dark solitary structures}

We also carried out a comprehensive study of vector short pulse dynamics in the anomalous dispersion regime at high pumping powers. By short pulse dynamics, we mean that we search for quasistationary temporal structures with temporal durations significantly shorter than the cavity round-trip time, which is $42 \mathrm{~ns}$. They are observed for pumping powers above $1 \mathrm{~W}$. According to the settings of the intracavity wave plates, orthogonal polarization components exhibit very different coupled dynamics. Figure 10 displays the shortest vector pulse structure found, which is a coupled dark-and-bright solitary wave circulating at the cavity round-trip frequency. Indeed, one polarization component displays a bright peak sitting on a noisy low background [see Fig. 10(b), gray (red) solid curve]. Whereas the orthogonal polarization component displays a dip on a noisy high cw background [see Fig. 10(c), black (blue) dotted curve]. The two temporal traces shown in Figs. 10(b) and 10(c) are recorded with a rise-time of 22 ps. The observed duration of the bright structure in Fig. 10(b) is $\sim 40 \mathrm{ps}$, and that of the dark structure Fig. 10(c) is $\sim 60$ ps. Optical spectra of both bright and dark pulse components of the vector temporal structures are presented in Fig. 10(a). Both spectra are centered around $1558 \mathrm{~nm}$. The FWHM of the bright temporal structure is $1.1 \mathrm{~nm}$ while that of the dark structure is $1.2 \mathrm{~nm}$. We note that the bright-dark localized dissipative structure compares qualitatively well with the strongly localized bright-dark soliton-like structure that was numerically obtained in Sec. II and displayed in Fig. 3.
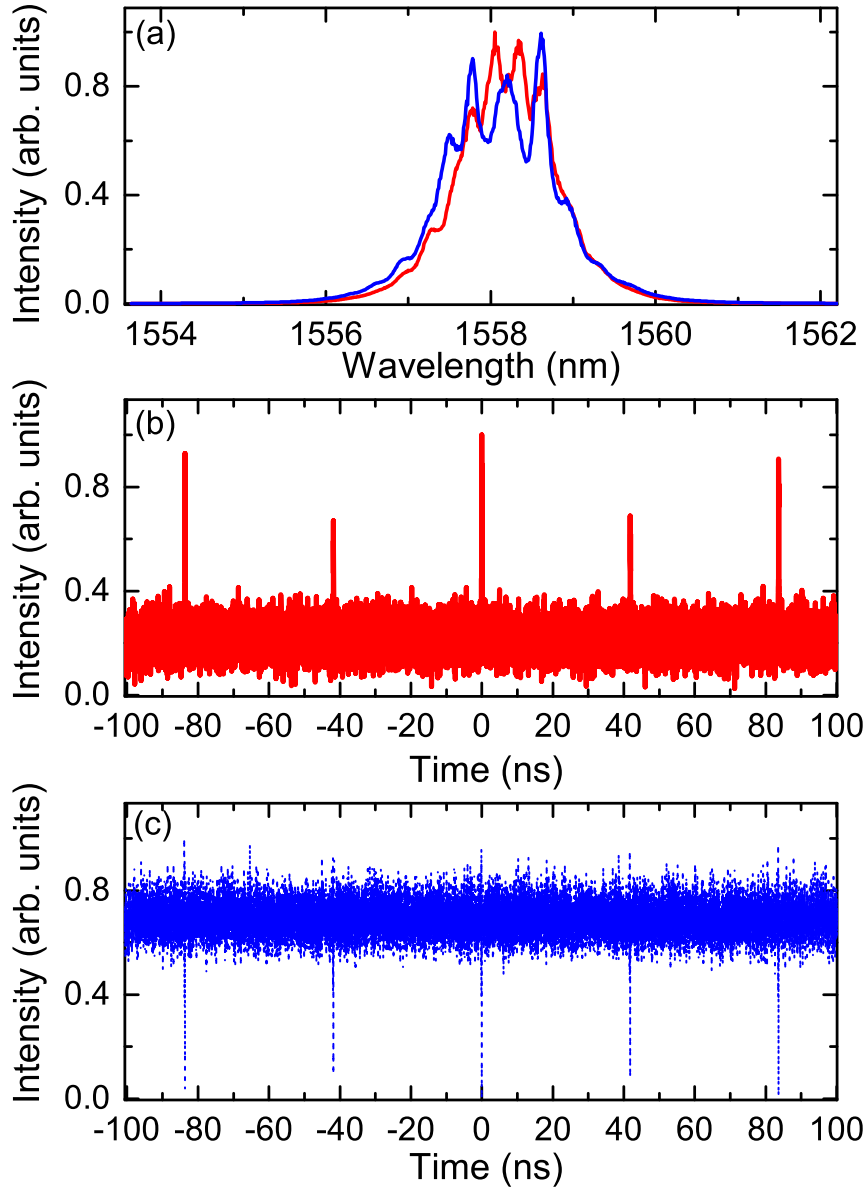

FIG. 10. (Color online) Short dark-bright coupled pulsations in the anomalous dispersion regime. (a) Optical spectra of the two orthogonal polarization states, and (b,c) their corresponding temporal traces (b). The gray (red online) solid curve in (a) is the spectrum corresponding to the gray (red online) solid temporal trace in (b). The black (blue online) dotted curve in (a) is the spectrum corresponding to the black (blue online) dotted temporal trace in (c).

\section{Polarization domain walls}

The detailed features of vector pulsations, such as the duration of the localized temporal structures, essentially depend on the orientation of the wave plates PC 1,2. It is possible to obtain long-lived polarization states once that the polarization switching transition has occurred, corresponding to relatively long, isolated, and periodic polarization domains separated by polarization domain walls. Figures 11 and 12 illustrate two distinct experimental observations of such PDW-like temporal dissipative structures. The second PDW-like structure, shown in Fig. 12, is obtained from the first structure of Fig. 11 after a slight adjustment (around $2^{\circ}$ ) of the quarter-wave plate (PC1) angle. Figures 11(a) and 12(a) present the optical spectra of both orthogonal polarization states corresponding to Figs. 11(b), 11(c), 12(b), and 12(c), respectively.

The temporal duration of the shorter polarization domain structure presented in Figs. 11(b) and 11(c) is around $1.5 \mathrm{~ns}$. Corresponding optical spectra, for both polarization components, appear in Fig. 11(a). The polarization domain duration increases to 2 ns [see Figs. 12(b) and 12(c)], while developing 

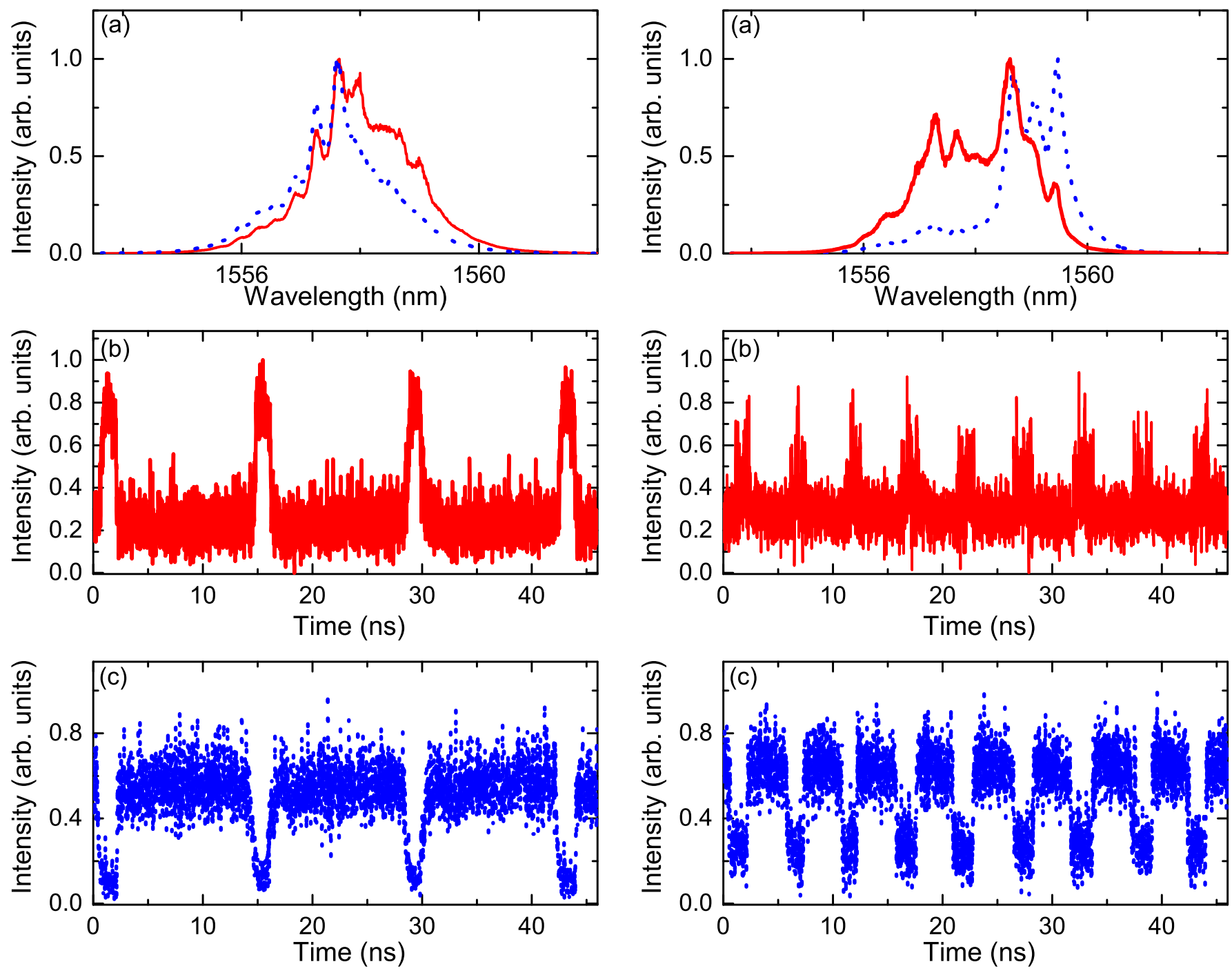

FIG. 11. (Color online) PDW-like temporal dissipative structures. (a) Optical spectra of the two orthogonal polarization states, and $(b, c)$ their corresponding temporal traces. Dark PDW-like structures are indicated by black (blue) dotted temporal traces and spectra; bright PDW-like structures are indicated by gray (red) solid temporal traces and spectra.

a nearly symmetric or square overall shape. Here, the central wavelength of the dark pulse is $1559 \mathrm{~nm}$ and its FWHM is $1.1 \mathrm{~nm}$ [see Fig. 12(c)]. The spectrum of the bright pulse is centered at $1558.0 \mathrm{~nm}$ and its width is broader (FWHM = $2 \mathrm{~nm}$ ). Optical spectra hardly change over the whole range of wave-plate orientations. Let us notice that Fig. 11 displays three PDW-like structures per cavity round-trip, whereas in the case of Fig. 12, there are eight such structures per round-trip. The experimental PDW-like vector structures of Figs. 11(b) and 11(c) and Figs. 12(b) and 12(c) are qualitatively reproduced by the polarization domains and domain walls which we numerically obtained in Figs. 1(c) and 2(c) by solving the CGLE (1). For appropriate settings of the PC angles, the localized square-waves as in Figs. 12(b) and 12(c) breakup into a set of vector multipulse solitary waves which have antiphase occurrence as in Figs. 7(c) to 8.

FIG. 12. (Color online) Eighth-harmonic PDW. (a) Optical spectra of the two orthogonal polarization states, and $(b, c)$ their corresponding temporal traces. Dark PDW-like structures are indicated by black (blue) dotted temporal traces and spectra; bright PDW-like structures are indicated by gray (red) solid temporal traces and spectra.

\section{E. Disordered antiphase dynamics}

To complete the observation of polarization dynamics, disordered polarization structures can also be obtained from the first dark-bright structure shown in Fig. 10. In fact, by tuning the PC angles, we observed disordered polarization evolutions that appear to be the signature of chaotic or intermittent vector solitary waves, whereas they still feature some degree of antiphase dynamics as far as the main pulse component appearing once per round-trip is concerned. The pattern of each polarization component is a succession of localized structures that are continuously created or annihilated in an irregular manner, as it is shown in Figs. 13(b) and 13(c). Nevertheless, the complex structure still exhibits quasiperiodicity at the cavity round-trip time. Within one round-trip time, the observed behavior appears to be in 

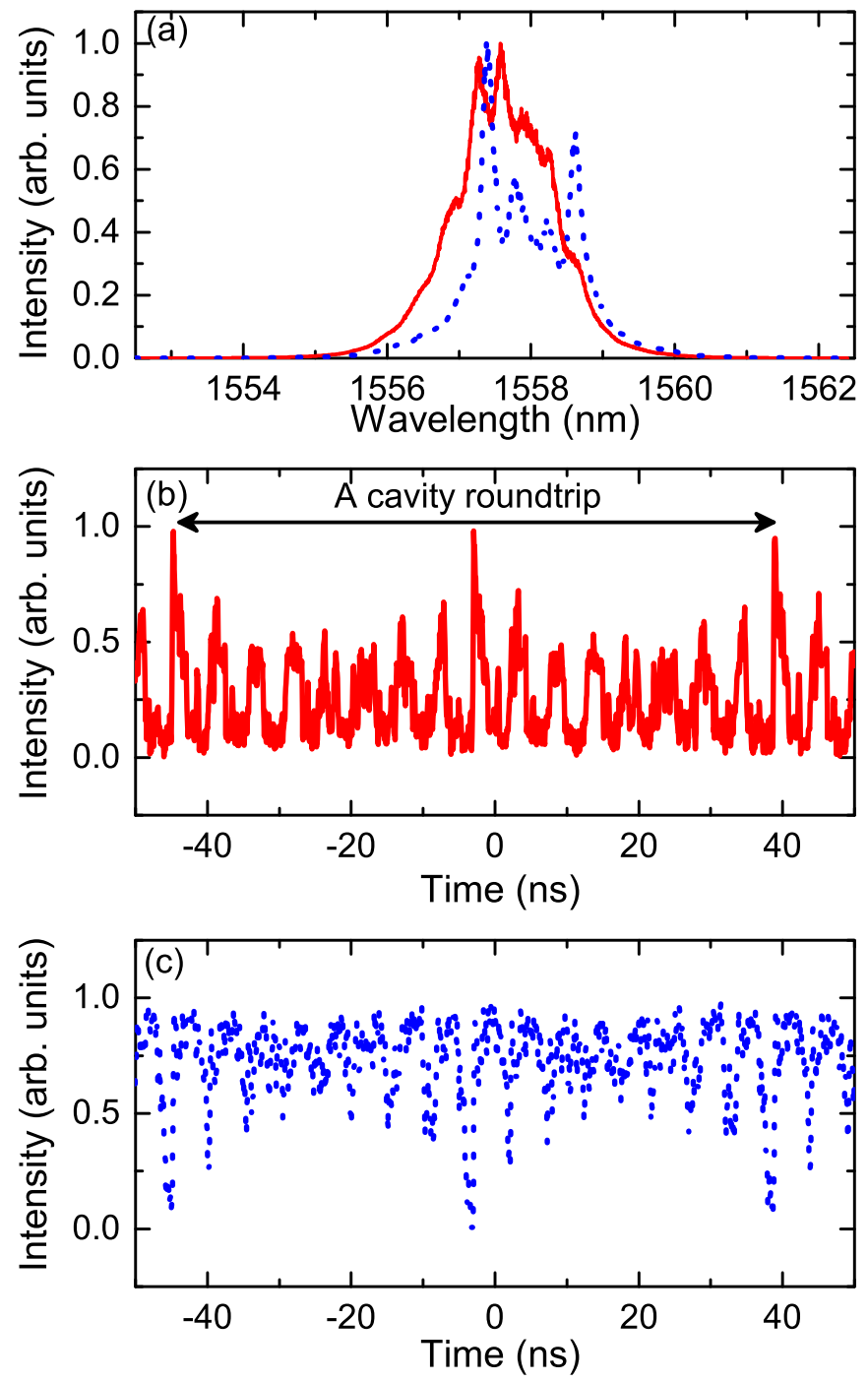

FIG. 13. (Color online) Disordered antiphase dynamics. (a) Optical spectra of the two orthogonal polarization states, and $(b, c)$ their corresponding temporal traces. Dark PDW-like structures are indicated by black (blue) dotted temporal traces and spectra; bright PDW-like structures are indicated by gray (red) solid temporal traces and spectra.

qualitative agreement with the numerical intensity profiles illustrated in Fig. 2(b).

\section{DISCUSSION AND CONCLUSION}

Our interpretation of the dynamics observed experimentally is the following. In an initial step, considering the similarity of our setup with that of Ref. [38], we conjecture that a RNGH-type instability is prone to convert part of the noisy, quasi-cw laser emission into nanosecond pulsations that repeat at the cavity fundamental frequency. Then, these pulsations are able to trigger subsequent nonlinear effects, which are mainly self- and crossed-Kerr and gain saturation nonlinearities, accompanied by the action of chromatic dispersion. This subsequent pulse shaping takes numerous cavity round-trips. In a vector fiber ring laser without polarizing components, the dominating dynamics is characterized by antiphase polarization dynamics separated by picosecond temporal polarization domain walls. For this type of dynamics, with nonlinear and dispersive lengths much longer than the cavity length, the use of a vector-distributed model appears to be justified.

Indeed, we have shown that the rich experimentally observed polarization dynamics may be qualitatively well reproduced in terms of solutions of the coupled one-dimensional complex Ginzburg-Landau equations. In particular, polarization cross-phase modulation and cross-gain saturation lead to the synchronization of spatiotemporal chaos into ordered states of temporally alternating orthogonal polarization domains. We have also shown that the control of polarization coupling may lead to generating stable lattices of antiphase soliton trains with high duty cycle at repetition rates of hundreds of $\mathrm{MHz}$, as well as coupled dark and bright soliton structures.

We may therefore conclude that vector fiber lasers provide an ideal testbed for testing the fundamental dynamics of chaos synchronization and control. From the practical side, the polarization dynamics of vector fiber lasers may find applications to chaos-based communication systems where decrypting of information is based on the synchronization of chaotic laser sources.

\section{ACKNOWLEDGMENTS}

This research was funded by iXCore Foundation, the Italian Ministry of University and Research (MIUR) (Grant No. 2012BFNWZ2), and by the Agence Nationale de la Recherche ("Solicristal" Project No. ANR-2010-BLANC-0417-01). We also thank the Labex ACTION program (ANR-11LABX-01-01).
[1] L. M. Pecora and T. L. Carroll, Phys. Rev. Lett. 64, 821 (1990).

[2] S. Boccaletti, J. Kurths, G. Osipov, D. L. Valladares, and C. S. Zhou, Phys. Rep. 366, 1 (2002).

[3] K. Wiesenfeld, P. Colet, and S. H. Strogatz, Phys. Rev. Lett. 76, 404 (1996).

[4] P. Colet and R. Roy, Opt. Lett. 19, 2056 (1994).

[5] J. Garcia-Ojalvo and R. Roy, Phys. Rev. Lett. 86, 5204 (2001).

[6] H. Chaté, Nonlinearity 7, 185 (1994).

[7] C. Lecaplain, Ph. Grelu, and S. Wabnitz, J. Opt. Soc. Am. B 30, 211 (2013).

[8] H. Sagaguchi, Phys. Scr. T 67, 148 (1996).
[9] A. Amengual, E. Hernández-Garcia, R. Montagne, and M. San Miguel, Phys. Rev. Lett. 78, 4379 (1997).

[10] R. Montagne and E. Hernández-Garcia, Phys. Lett. A 273, 239 (2000)

[11] E. Hernández-Garcia, M. Hoyuelos, P. Colet, M. San Miguel, and R. Montagne, Int. J. Bifurcation Chaos 09, 2257 (1999).

[12] M. van Hecke, C. Storm, and W. van Saarloos, Physica D 134, 1 (1999).

[13] H. Haken, Synergetics, An Introduction (Springer, Berlin, 1978).

[14] P. Manneville, Dissipative Structures and Weak Turbulence (Academic, San Diego, CA, 1990). 
[15] M. C. Cross and P. C. Hohenberg, Rev. Mod. Phys. 65, 851 (1993).

[16] S. Kogelman and R. C. DiPrima, Phys. Fluids 13, 1 (1970).

[17] K. Stewartson and J. T. Stuart, J. Fluid Mech. 48, 529 (1971).

[18] Y. Kuramoto and T. Yamada, Prog. Theor. Phys. 56, 679 (1976).

[19] N. Bekki, J. Phys. Soc. Jpn. 50, 659 (1981).

[20] Pattern Formation in Liquid Crystals, edited by A. Buka, L. Kramer (Springer, New York, 1996).

[21] Physics of Biological Systems, edited by H. Flyvbjerg, J. Hertz, M. H. Jensen, O. G. Mouritsen, and K. Sneppen (Springer, Heidelberg, 1997).

[22] M. San Miguel, Phys. Rev. Lett. 75, 425 (1995).

[23] J. M. Soto-Crespo, N. N. Akhmediev, B. C. Collings, S. T. Cundiff, K. Bergman, and W. H. Knox, J. Opt. Soc. Am. B 17, 366 (2000).

[24] Ph. Grelu and N. Akhmediev, Nat. Photon. 6, 84 (2012).

[25] Q. L. Williams, J. Garcia-Ojalvo, and R. Roy, Phys. Rev. A 55, 2376 (1997).

[26] H. Zhang, D. Y. Tang, L. M. Zhao, and X. Wu, Phys. Rev. B 80, 052302 (2009).

[27] H. Zhang, D. Y. Tang, L. M. Zhao, and R. J. Knize, Opt. Express 18, 4428 (2010).

[28] H. Y. Wang, W. C. Xu, W. J. Cao, L. Y. Wang, and J. L. Dong, Laser Phys. 22, 282 (2012).

[29] M. Haelterman and A. P. Sheppard, Opt. Lett. 19, 96 (1994).

[30] M. Haelterman and A. P. Sheppard, Phys. Rev. E 49, 4512 (1994).
[31] K. Otsuka, P. Mandel, S. Bielawski, D. Derozier, and P. Glorieux, Phys. Rev. A 46, 1692 (1992).

[32] P. Le Boudec, C. Jaouen, P. L. François, J.-F. Bayon, F. Sanchez, P. Besnard, and G. Stéphan, Opt. Lett. 18, 1890 (1993).

[33] A. J. Poustie, Opt. Lett. 20, 1868 (1995).

[34] F. Sanchez and G. Stephan, Phys. Rev. E 53, 2110 (1996).

[35] E. Louvergneaux, F. Rogister, and P. Glorieux, Phys. Rev. Lett. 99, 263901 (2007).

[36] F. Prati, E. M. Pessina, G. J. de Valcárcel, and E. Roldán, Opt. Commun. 237, 189 (2004).

[37] E. M. Pessina, G. Bonfrate, F. Fontana, and L. A. Lugiato, Phys. Rev. A 56, 4086 (1997).

[38] T. Voigt, M. O. Lenz, F. Mitschke, E. Roldán, and G. J. de Valcárcel, Appl. Phys. B 79, 175 (2004).

[39] Rare Earth Doped Fiber Lasers and Amplifiers, edited by M. J. F. Digonnet (Marcel Dekker, New York, 1993).

[40] Y. Sun, J. L. Zyskind, and A. K. Srivastava, IEEE J. Sel. Top. Quantum Electron. 3, 991 (1997).

[41] A. Zaviyalov, Ph. Grelu, and F. Lederer, Opt. Lett. 37, 175 (2012).

[42] F. Brunet, Y. Taillon, P. Galarneau, and S. LaRochelle, J. Light. Technol. 23, 2131 (2005).

[43] R. Montagne, E. Hernández-Garcia, and M. San Miguel, Phys. Rev. Lett. 77, 267 (1996).

[44] M. van Hecke, Phys. Rev. Lett. 80, 1896 (1998).

[45] N. R. Pereira and L. Stenflo, Phys. Fluids 20, 1733 (1977). 